\title{
Multiple completions primed by occlusion patterns
}

Rob J van Lier, Emanuel $L \mathrm{~J}$ Leeuwenberg, Peter $A$ van der Helm

Nijmegen Institute for Cognition and Information (NICl), University of Nijmegen, PO Box 9104 , 6500 HE Nijmegen, The Netherlands

Received 28 July 1993, in revised form 4 October 1994

\begin{abstract}
There is a strong tendency to complete a partly occluded shape. Two types of pattern completion, global and local, are frequently reported. By means of the primed-matching paradigm, it has previously been shown that global completions are prevalent for stimuli in which regularity is abundantly present. In our study the primed-matching paradigm is applied to such stimuli in order to find out whether the rival local completion is generated as well. Therefore anomalous completions are added to the experimental design. Priming effects both on global and on local completions are compared with priming effects on those anomalous completions. The data indeed suggest that the occlusion patterns evoked not only a global but also a local completion.
\end{abstract}

\section{Introduction}

Usually objects occlude parts of themselves and parts of other objects. Yet we do not experience a fragmented world. Apparently the visual system completes partly occluded objects, rapidly and unconsciously. This completion phenomenon has been studied a number of times during past years. Without claiming completeness let us mention a few of these studies: Boselie 1988, 1994; Boselie and Wouterlood 1989; Buffart et al 1981, 1983; Dinnerstein and Wertheimer 1957; Gerbino and Salmaso 1987; Kanizsa and Gerbino 1982; Kellman and Shipley 1991; Sekuler 1994; Sekuler and Palmer 1992; Sekuler et al 1994; Takeishi et al 1993; van Lier et al 1994, 1995; Wouterlood and Boselie 1992. Although these studies were focused on various aspects of pattern completion, they all dealt with the perceived form of the occluded shape. The topic is illustrated in figure 1. Three interpretations of the left pattern are shown on the right. A in figure 1 represents the 'mosaic' interpretation in which an L-shaped form is juxtaposed to a square, whereas $B$ represents an occlusion interpretation, in which the background figure is completed into a rectangle. Obviously many more completions are possible but unlikely, such as the anomalous completion, $\mathrm{C}$, in figure 1 . In this paper we will focus only on patterns that evoke occlusion interpretations.

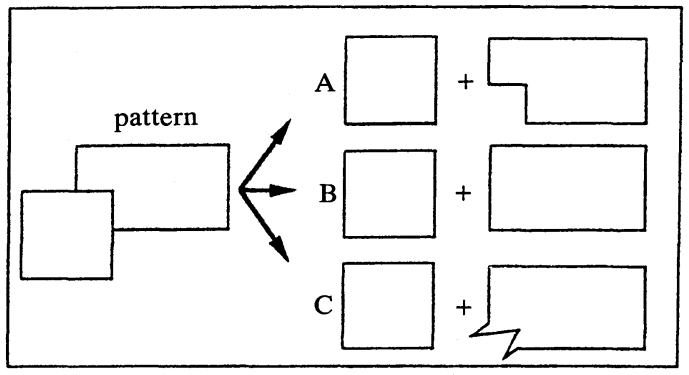

Figure 1. Three interpretations of the pattern on the left-hand side are given. A represents a 'mosaic' interpretation, $\mathrm{B}$ the preferred completion, and $\mathrm{C}$ an anomalous completion. 


\subsection{Global and local completions}

Two types of pattern completion often appear to be relevant: completions based on global simplicity and completions based on local simplicity (see figure 2). The completion $\mathrm{A}$ in figure 2 is based on global simplicity. That is, the completed background shape reveals a maximum of regularities, which is reflected by, for example, its number of axes of symmetry. The completion B in figure 2 is based on local simplicity. This local simplicity corresponds with the 'good continuation' of the visible contours of the background shape until they meet.

During past decades, arguments for the prevalence of either global or local completions in visual occlusion have been put forward, often related to certain pattern properties. In general, 'local' theories rely strongly on the impact of local cues, such as specific discontinuities of contours at points of occlusion. The Gestalt principle of good continuation is an important aspect in these theories. For example, in the completion model advocated by Wouterlood and Boselie (1992), a completion based on good continuation of the occluded contours is predicted whenever specific junction types or combinations of those junctions occur. Although Boselie (1994) demonstrated that local aspects also have a strong influence in regular patterns, their model so far is restricted to irregular patterns. The local-completion theory of Kellman and Shipley (1991) may be regarded as a formalisation of the good-continuation principle. They argued that completion is reached by a smooth continuation of contours at points of occlusion. Consequently, their model disregards the influence of pattern regularities in predicting perceived completions. In contrast, 'global' theories take into account the regularities of the whole pattern in predicting completions. The Gestalt principle of Prägnanz plays an important role in these theories. Buffart et al (1981, 1983) combined a coding scheme with the global-minimum principle, stating that the simplest completion will be perceived. Despite the initial success of their approach, various patterns have been constructed for which the perceived shape is not the simplest (eg Boselie 1988; Kanizsa 1985; Rock 1983).

The ongoing controversy between global and local theories led some researchers to the conclusion that both global and local aspects play a role in pattern completion. Boselie (1988) argued that in a case where two locally simplest interpretations can be made, the globally simplest will be preferred. Recently, Sekuler (1994) gave a qualitative description of a completion model in which the saliency of global and local completions depends on the number and orientation of the axes of symmetry. We demonstrated (van Lier et al 1995) a relation between the degree of occlusion and the saliency of a global completion. Moreover, we presented (van Lier et al 1994) a

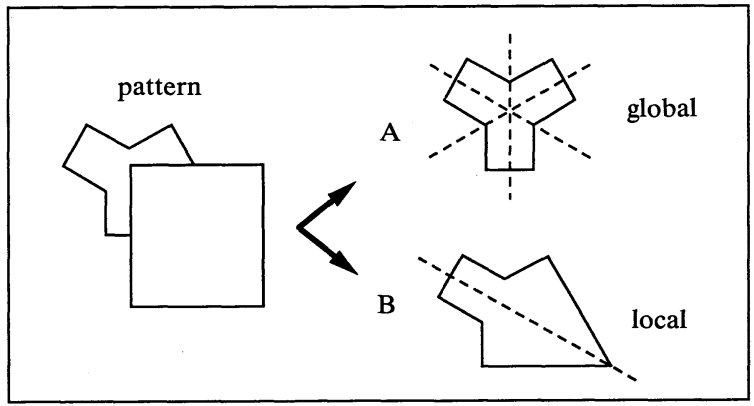

Figure 2. Two completions of the pattern on the left-hand side are given. A represents the global completion and B the local completion. The global completion is based on a tendency towards a maximum of regularity of the completed shape, while the local completion is based on 'good continuation' of the contours of the background shape. In the present case the global shape has three axes of symmetry and the local completion one axis of symmetry. 
completion model in which regularities within and between shapes as well as the degree of occlusion are quantified. The model predicts completions in which the sum of these quantified aspects is minimal. Other integrative approaches such as that of Shimaya (1994) also indicate that both global and local aspects are crucial with respect to the perceived interpretation.

\subsection{Single versus multiple completions}

In this paper, however, we do not go into detail on the question of which specific pattern properties support global or local completions, nor do we elaborate on differences between existing theories with respect to the specific definitions of global and local completions. As indicated already, we simply will consider completions to be global if the completed shape reveals a maximum of symmetry and local if it is reached by linear good continuation of the occluded contours. Given the different types of completions, we will deal here with an aspect of the completion process by questioning whether the perceptual system processes either global or local completions, or both global and local completions. Herbart (1850) has already suggested that the degree to which one interpretation is preferred to another is the result of competition between several possible candidates. In line with this tradition, Mens and Leeuwenberg (1988) demonstrated the hidden presence of alternative interpretations for certain visual patterns. Furthermore, it was argued by Buffart et al (1983) that, in the case of visual completion, the relative dominance of completions depends on their relative complexity. Also, in Sekuler's (1994) qualitative model on pattern completion the generation of multiple completions is suggested as it concerns both a global and a local completion-processing module. In van Lier et al (1995) we discussed the plausibility of competition between global and local completions, using the results of a spontaneous pattern-interpretation task, in which subjects had to draw the preferred completion, and the results of a simultaneous-matching task, partly adopted from Gerbino and Salmaso (1987). The competitive aspect between completions gained support from the finding that the strength of a specific global completion depends on the strength of the local completion. This interdependence already suggested the processing of both global and local completions. Here we aim to converge evidence for the generation of multiple completions by means of a more straightforward test: the primed-matching paradigm.

\section{Priming pattern completions}

Recently, the primed-matching paradigm was introduced in the research field of pattern completion by Sekuler and Palmer (1992). This paradigm turned out to be a useful tool to assess a subject's perceptual representation. The essence of the primedmatching paradigm lies in a comparison between the effects of different primes on an immediately following matching task. In that task, subjects had to judge as fast as possible whether two simultaneously displayed shapes are equal or not. The inferences drawn from results obtained by the primed-matching paradigm are based on previous findings that the response time to identical items is a function of the representational similarity between the items and the prime (cf Beller 1971; Rosch 1975a, 1975b; Sekuler and Palmer 1992). High similarities appeared to facilitate the response. Figure 3 shows the kind of patterns of the study of Sekuler and Palmer (1992).

In an experimental trial, one of the primes on the left can be succeeded by one of the test pairs on the right. According to Sekuler and Palmer's findings a 'same' response on test pair 1 (two complete circles) requires less response time after prime $A$ (the circle) than after prime $B$ (the incomplete circle). Correspondingly, the 'same' response on test pair 2 (two incomplete circles) will be faster after prime B than after prime $A$. The crucial prime now is the ambiguous prime $C$, which can be 
interpreted either as a complete circle behind a square, or as an incomplete circle juxtaposed to a square. It appeared that after a prime duration of a few hundred milliseconds, prime $\mathrm{C}$ and prime $\mathrm{A}$ had the same facilitating effects on test pair 1 . Moreover, prime $\mathrm{C}$ did not facilitate responses on test pair 2. These results provided strong indication that prime $\mathrm{C}$ was interpreted as a case of visual occlusion in which the background shape was completed into a full circle. The variation of prime duration enabled Sekuler and Palmer to follow the time course of the completion process. Their results suggest that the completion process is finished after about $200 \mathrm{~ms}$.

The occlusion patterns in the study by Sekuler and Palmer (1992) were such that the global completion had the same shape as the local completion. Recently, Sekuler et al (1994) used the primed-matching paradigm in order to investigate patterns whose global completions were different from the local completions. In that study, occlusion patterns were used for which the global completion yielded more than one axis of symmetry. For priming durations of $150 \mathrm{~ms}$ up to $1000 \mathrm{~ms}$, the effects of primes with occluded shapes (the occlusion prime) were compared with those of primes having either an unoccluded globally completed shape or an unoccluded locally completed shape. It appeared that the effects of the occlusion prime were more similar to the effects of primes with the unoccluded globally completed shape. Sekuler et al concluded that completions of occlusion patterns were not dominated by a local process, at least not for their set of patterns. That is, at early stages of the completion process, the occluded shapes are completed in such a way that the complete shapes possess a maximum of symmetry. In another recent study Sekuler (1994) additionally showed that completion depends on the number and orientation of the axes of symmetry. Sekuler concluded that, in contrast with the results on the highly regular patterns, local completion processes may dominate in case of limited pattern regularities.

In the above studies, priming effects of occlusion patterns were compared with priming effects of patterns in which either the global or the local completion was completely visible. In that way it could be investigated whether the global or the local completion was dominant. We pose another question here: does the dominance of one type of completion imply the absence of the other type of completion or might the latter completion be generated as well? In view of the primed-matching paradigm we question whether these nondominant completions are not primed at all or whether they are primed weakly. Indeed, if the latter is the case it supports the notion that both global and local completions are generated by the perceptual system. Our goal

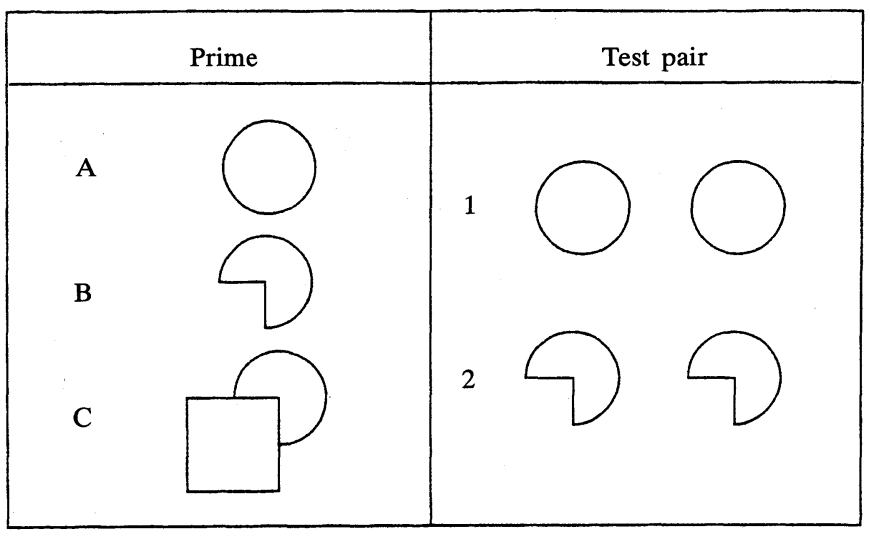

Figure 3. Primes and test pairs as can be used in the primed-matching paradigm. After prime durations of about $200 \mathrm{~ms}$, primes $\mathrm{A}$ and $\mathrm{C}$ have the same facilitating effects on test pair 1 , whereas primes $B$ and $C$ have different effects on test pair 2 . 
here is to investigate this perceptual relevance of global and local completions for a specific occlusion pattern. Microgenetic processing aspects dealing with questions such as whether these completions are generated in parallel or sequentially or whether completions are concurrently or alternately present are different issues and lie beyond the scope of this study.

In the following experiment we use patterns in which regularity is abundantly present. Anomalous completions of the occlusion prime are added to the experimental design. As a result, local completions will not only contrast with global completions, but also with those anomalous completions. The latter comparison will be crucial with respect to the question of whether the local completions are generated as well.

\section{Experiment 1}

\subsection{Subjects}

Thirty students of the University of Nijmegen participated in the experiment. All participants received course credits.

\subsection{Stimuli}

We restricted ourselves to a small set of patterns in which global completions are prevalent. All patterns stem from our previous research (van Lier et al 1995) (see figure 4). Notice that the globally completed shapes either have at least three axes of symmetry or are at least threefold rotation symmetric. For each pattern three completions are considered: a global completion, a local completion, and an anomalous completion. Each pattern gives rise to five different primes and six different test pairs. An example is given in figure 5. In this figure all primes and test pairs for the first pattern of figure 4 are shown. Prime $\mathrm{A}$ is the occlusion prime, $\mathrm{B}$ the global foreground prime, $\mathrm{C}$ the local foreground prime, $\mathrm{D}$ the anomalous foreground prime, and E represents the no-prime condition. The six test pairs consist of the following combinations of shapes: global/global, local/local, anomalous/anomalous, global/local, anomalous/global, local/anomalous. Each combination of prime and test pair represents a stimulus presentation. Thus, each occlusion pattern of figure 4

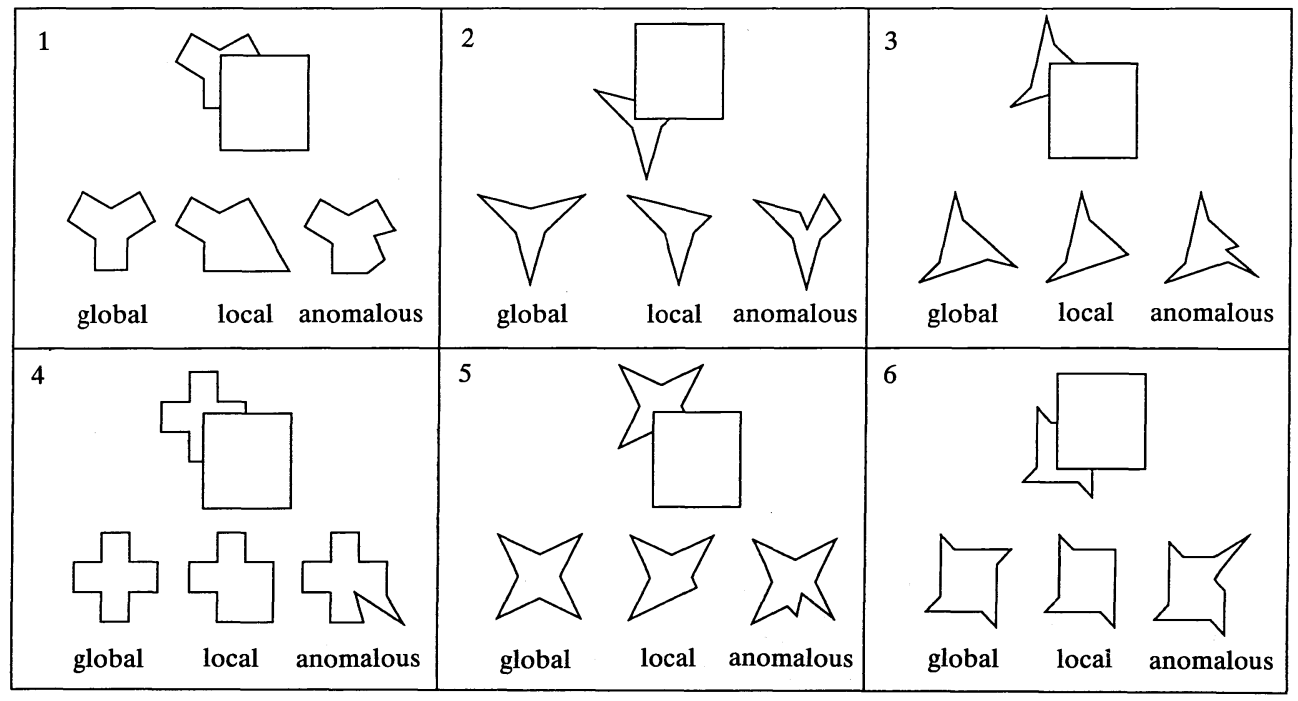

Figure 4. All occlusion patterns that served as an occlusion prime in experiment 1 are depicted. Beneath each pattern three completions are given: global, local, and anomalous, respectively. 
induces $5 \times 6=30$ stimuli, which will be referred to as a 'stimulus subset'. Because there are six stimulus subsets, there are $6 \times 30=180$ stimuli.

Like Sekuler and Palmer (1992), Sekuler et al (1994), and Sekuler (1994), we will only consider response times on the test pairs with identical shapes in the analysis, because priming effects may be expected only on pairs with identical shapes (cf Beller 1971). In the following, test pair 1, 2, and 3 of figure 5 will be referred to as the global, the local, and the anomalous test pair, respectively. A foreground prime succeeded by a test pair with shapes that are both identical to the shape of the prime will be referred to as a congruent foreground prime. For example, if prime B in figure 5 (the global foreground prime) is followed by the global test pair it will be referred to as a congruent foreground prime. The same holds for the combinations (prime $\mathrm{C}$ and test pair 2), and (prime $\mathrm{D}$ and test pair 3). A foreground prime which is followed by a test pair with identical shapes, but different from the prime, will be referred to as an incongruent foreground prime. For example, if prime B in figure 5 is followed by test pair 2 or 3 , it will be referred to as an incongruent foreground prime. The same holds for the combinations (prime $C$ and test pair 1), (prime $C$ and test pair 3), (prime D and test pair 1), and (prime D and test pair 2).

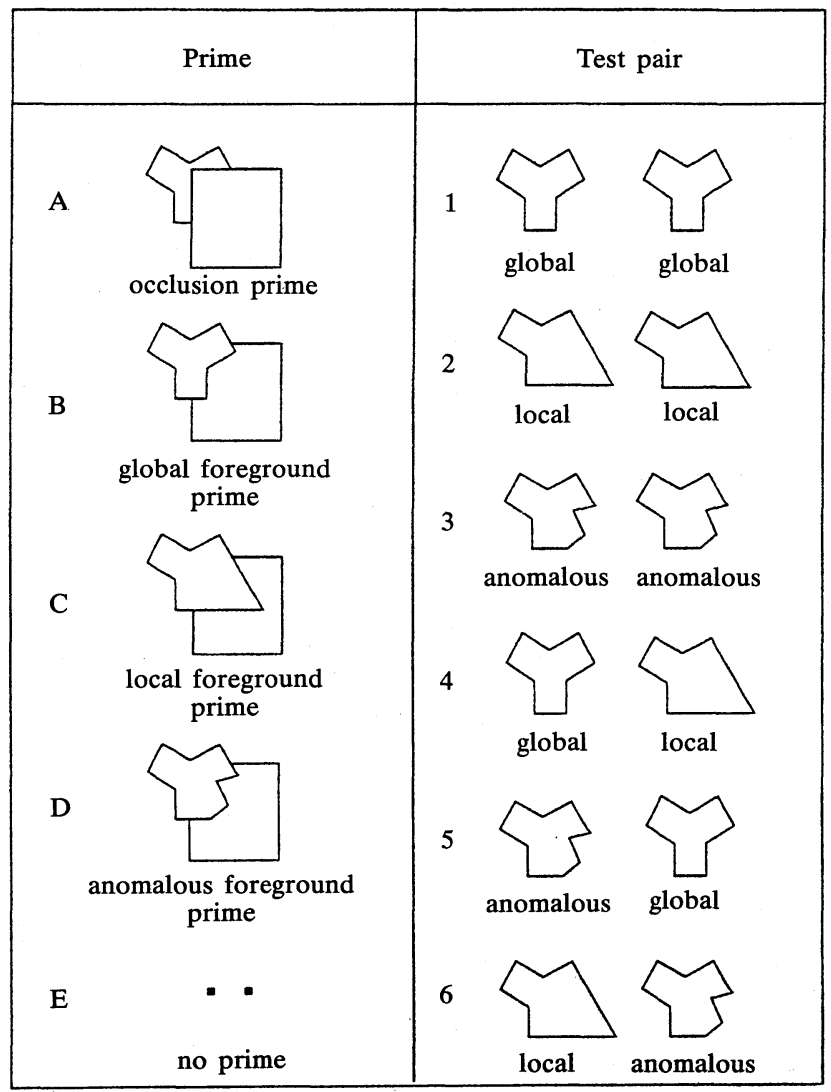

Figure 5. In the design of experiment 1 each occlusion pattern of figure 4 gives rise to five different primes and six different test pairs. Each combination of prime and test pair represents a stimulus in the experiment. 


\subsection{Procedure}

The stimuli were generated on a computer monitor with a viewing distance of $1 \mathrm{~m}$. The priming pattern was visible within a visual angle of $3 \mathrm{deg}$. The test pair was visible within a visual angle of $8 \mathrm{deg}$. In figure 6 the sequence of events and spatial layout in one trial is shown.

First a fixation point was shown at the centre of the screen for $500 \mathrm{~ms}$. After this period the screen was empty for $50 \mathrm{~ms}$. Subsequently the prime appeared. Prime duration was $750 \mathrm{~ms}$, in accordance with experiment 1 of Sekuler and Palmer (1992). According to the results of Sekuler and Palmer (1992) and Sekuler et al (1994) the completion process is finished within that period. $17 \mathrm{~ms}$ after the prime disappeared the test pair was shown. One of the two shapes of the test pair was positioned to the left and the other to the right of the previously shown prime. In addition a rectangle appeared in the centre top of the screen in order to avoid a severe weakening of the priming effect due to an apparent motion from the rectangle accompanying the relevant prime shape to one of the shapes in the test pair (see also Sekuler and Palmer 1992). The test pair remained on the screen until a response was given. Subjects responded by pushing one of two buttons (yes/no) in order to answer whether or not both test shapes were equal. Responses were measured to the nearest millisecond. As in the experiments of Sekuler and Palmer (1992), subjects received visual feedback on their response time. If an answer was incorrect this was reported to the subject. Incorrectly answered stimuli were presented once more at a later stage in the experiment. The order of presentation was randomised for each subject.

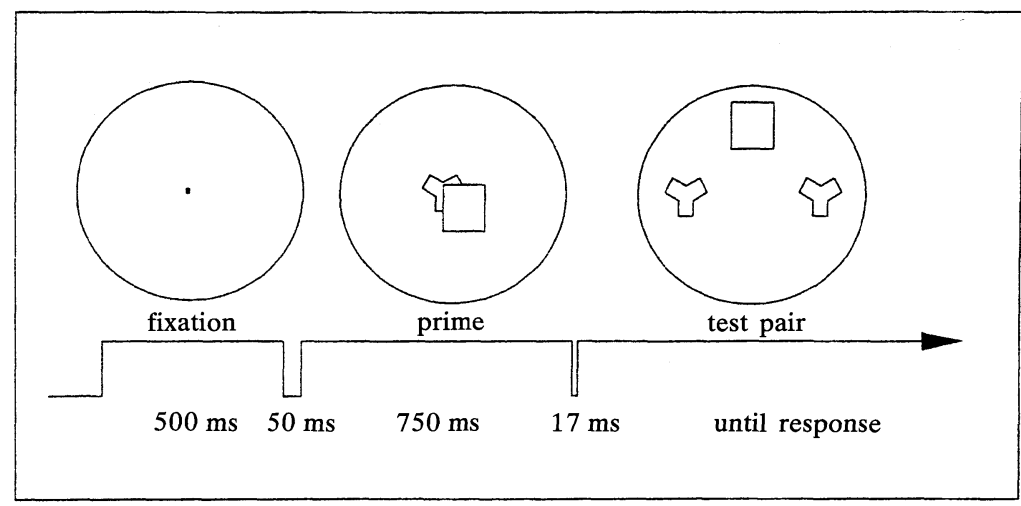

Figure 6. The spatial and temporal layout of the experimental procedure.

\subsection{Results}

Remember that only response times stemming from correct 'yes' responses are considered. The mean response times for each prime $\times$ test pair combination are plotted in figure 7. In this figure the test pairs are plotted on the horizontal axis. The various primes are represented by different symbols. For each test pair, homogeneous subsets according to Tukey's HSD procedure $(p<0.05)$ are indicated by ovals.

In figure 7 , it can be seen that congruent foreground primes, for example the global foreground prime followed by the global test pair, evoke the fastest response times. This is exactly what is expected on the basis of the assumptions underlying the primed-matching paradigm. Accordingly, the incongruent foreground primes evoke relatively slow response times. For example, the response time on the global test shapes is the slowest after the local foreground prime, whereas the response time on the local test shapes is the slowest after the global foreground prime. The issue here is the priming effect of the occlusion prime on the test pairs. The results of Tukey's 
HSD test on the response times already indicate the varying effects of the occlusion pattern on the three test pairs. In the following we evaluate the priming effects of the occlusion prime. This priming effect is supposed to reveal the strength of the completion of the occlusion prime.
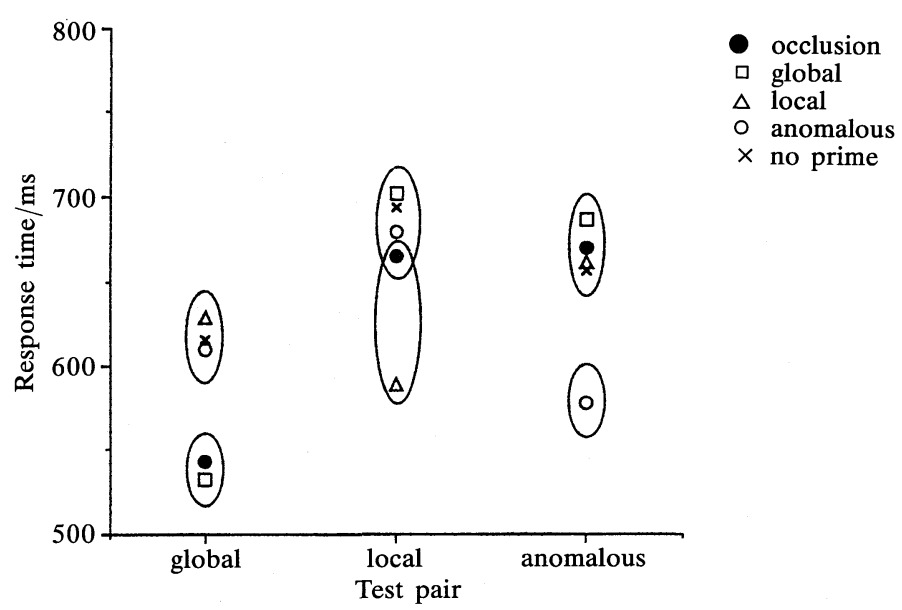

Figure 7. The mean response times on all prime $\times$ test pair combinations in experiment 1 . On the horizontal axis the test pairs are given. The primes are represented by different symbols. For each test pair, homogeneous subsets according to Tukey's HSD procedure $(p<0.05)$ are indicated by ovals.

\subsection{Analyses}

Sekuler (1994) and Sekuler et al (1994) defined the priming effect for a given prime by means of the difference in response time on global and local test pairs after the prime, minus this difference after no prime. The response times after no prime served as a 'baseline', as the inclusion of these response times accounted for the possibility that subjects responded faster to global test pairs than to local test pairs, even after no prime. In this way it could be tested whether the priming effects of the occlusion prime were more similar to those of the global foreground prime or to those of the local foreground prime. Our design differs from that of Sekuler and Sekuler et al in that anomalous completions are also included. We therefore reconsider the priming effect. In the present analyses we will compare the priming effects of the occlusion prime on the global, local, and anomalous test pairs. Below we give two analyses, thereby defining the priming effect in two different ways.

3.5.1 Analysis 1. For each test pair, the response times after no prime, the occlusion prime, and the congruent foreground prime will be considered. If the occlusion prime is completed in such a way that it is the same as the test shapes, then we expect the response time after the occlusion prime to be shorter than the response time after no prime. Also, we expect that the response time after the occlusion prime will approach the response time after the congruent foreground prime. As both the response time after no prime and the response time after the congruent foreground prime may be regarded as a baseline value we will take them both into account, by taking the mean of these response times. The priming effect of the occlusion prime on a given test pair will be determined by the difference between this mean baseline value for a given test pair and the response time after the occlusion prime on that test pair:

$$
\mathrm{PE}(\mathrm{OP} \mid \mathrm{TP})=\overline{\mathrm{RT}\{(\mathrm{NP} \mid \mathrm{TP}),(\mathrm{CFP} \mid \mathrm{TP})\}}-\mathrm{RT}(\mathrm{OP} \mid \mathrm{TP}),
$$

where $\mathrm{PE}$ is the priming effect, OP occlusion prime, TP test pair, RT response time, NP no prime, and CFP congruent foreground prime. 
In figure 8, the mean PE values for experiment 1 are shown. An ANOVA for repeated measurements with $\mathrm{PE}$ as the dependent variable has been performed. The main effect of test pair was significant $\left(F_{2,58}=11.24, p<0.001\right)$. There was no significant interaction between test pair and the six stimulus subsets $\left(F_{10,290}=1.32\right)$. In addition, one tailed $t$-tests were performed on the mean priming effects; the results are the following: $\quad \mathrm{PE}(\mathrm{OP} \mid$ global $)>\mathrm{PE}(\mathrm{OP} \mid$ local $), \quad t=2.57 \quad(p<0.01) ; \quad \mathrm{PE}(\mathrm{OP}||$ global $)>$ $\mathrm{PE}(\mathrm{OP} \mid$ anomalous $), \quad t=4.87 \quad(p<0.001) ; \quad \mathrm{PE}(\mathrm{OP} \mid$ local $)>\mathrm{PE}(\mathrm{OP} \mid$ anomalous $)$, $t=1.97(p<0.05)$.

Discussion. The priming effect of the occlusion prime on the local shapes lies between the priming effects on the global and anomalous shapes. The fact that the priming effects of the occlusion prime on both the global and the local test shapes are significantly higher than the priming effect on the anomalous test shapes supports the notion that besides the global completion, the local completion is generated.

In spite of the clear results on the mean data it is expedient to take a closer look at the tendencies within each stimulus subset. In figure 9 the priming effect within each stimulus subset is depicted. In five out of the six cases, the priming effect follows the tendency of the mean data, although differences between the priming effects on local and anomalous test shapes are fairly small in stimulus subsets 2 and 6 . The priming effect within stimulus subset 4 reveals a clear exception with respect to the mean data. Within this stimulus subset, the priming effect on the anomalous shapes appears to be almost the same as the priming effect on the global shapes. Does this mean that the occlusion prime is perceptually completed in an anomalous way, exactly as the one in figure 4? This seems to be highly unlikely. Apparently we have to preserve some caution with respect to the defined priming effect.

It can be questioned whether the priming effect of the occlusion prime, as defined in the present analysis, is solely caused by the perceptual completion of the occluded shape in the occlusion prime. Priming effects also could stem from the similarity between the visible part of the occlusion prime and the test shape. So, because of that

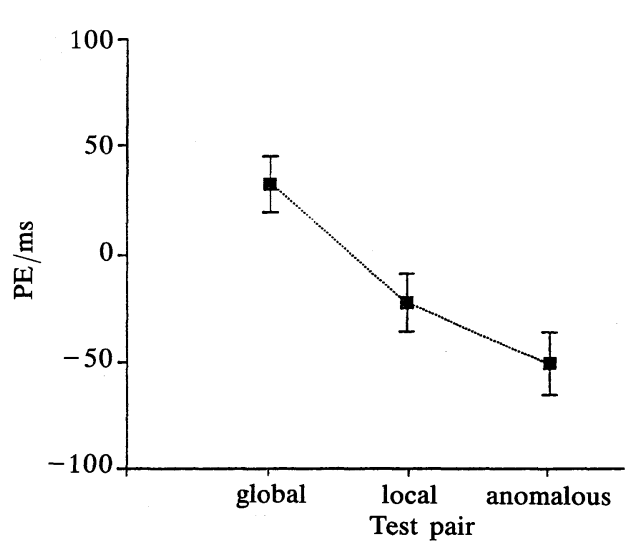

Figure 8. Experiment 1. The mean priming effect $(\mathrm{PE})$ according to analysis 1 . In this and subsequent figures, vertical bars represent \pm 1 SEM.

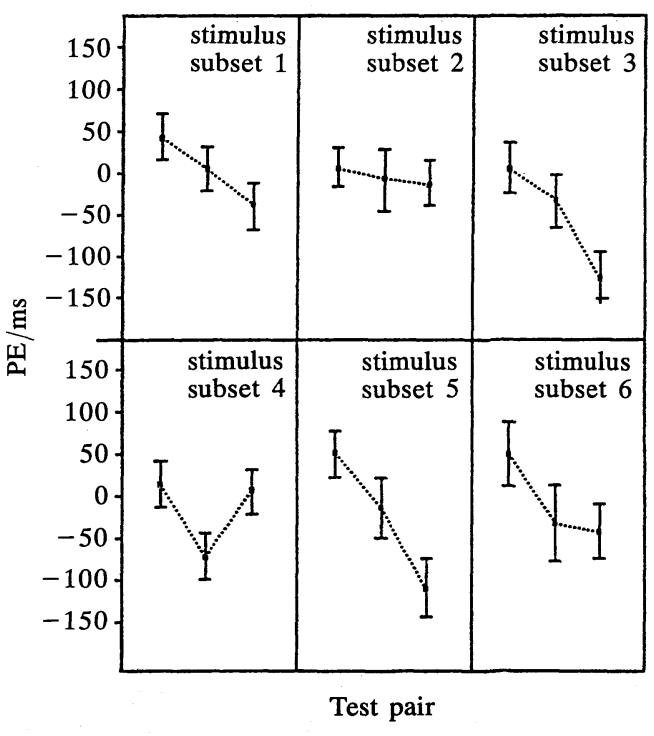

Figure 9. Experiment 1. The priming effect (PE) according to analysis 1 is plotted for each stimulus subset. The sequence of test pairs from left to right is: global, local, anomalous. 
part, even incongruent foreground primes might facilitate responses on a specific test pair. If the shorter response times after the occlusion prime are due to completion, they have to be different from the response times after the incongruent foreground primes. In the following analysis the priming due to this similarity is accounted for.

3.5.2 Analysis 2. Just like the occlusion prime, the incongruent foreground primes have the visible part of the occluded shape in the occlusion prime in common with the test shape. Therefore, in the present analysis we will consider the response times on the set of primes ( $\mathrm{S}$ ) consisting of the incongruent foreground primes and the occlusion prime as a baseline value. In addition, the priming effect of the occlusion prime will be conceived as the distinctiveness of the occlusion prime with respect to $S$. This priming effect, $\mathrm{PE}^{\prime}$, will be defined by the following expression:

$$
\mathrm{PE}^{\prime \prime}(\mathrm{OP} \mid \mathrm{TP})=\frac{\overline{\mathrm{RT}(\mathrm{S} \mid \mathrm{TP})}-\mathrm{RT}(\mathrm{OP} \mid \mathrm{TP})}{\sigma_{[\mathrm{RT}(\mathrm{S} \mid \mathrm{TP})]}} .
$$

The numerator, being the difference between the mean response time of $S$ and the response time after the occlusion prime, expresses the relative facilitation caused by the occlusion prime. This relative facilitation is normalised by the variation $(\sigma)$ between the response times: the greater the difference between the response times evoked by the incongruent foreground primes and the occlusion prime, the less distinctive the response time of the occlusion prime, and therefore the lower the priming effect.

If we consider all data, the $\mathrm{PE}^{\prime}$ values of the occlusion prime on the different test pairs are: $\mathrm{PE}^{\prime}(\mathrm{OP} \mid$ global $)=0.4188 ; \quad \mathrm{PE}^{\prime}(\mathrm{OP} \mid$ local $)=0.1598 ; \quad \mathrm{PE}^{\prime}(\mathrm{OP} \mid$ anomalous $)$ $=-0.0146$. The mean $\mathrm{PE}^{\prime}$ values are plotted in figure 10. An ANOVA for repeated measurements with $\mathrm{PE}^{\prime}$ as the dependent variable was performed. The main effect of test pair was significant $\left(F_{2,58}=14.56, p<0.001\right)$. There was no significant interaction between test pair and the six stimulus subsets $\left(F_{10,290}=0.89\right)$. $t$-tests performed on the mean priming effects reveal the following results: $\mathrm{PE}^{\prime}(\mathrm{OP} \mid \mathrm{global})>$ $\mathrm{PE}^{\prime}(\mathrm{OP} \mid$ local $), t=3.14,(p<0.01) ; \mathrm{PE}^{\prime}(\mathrm{OP} \mid$ global $)>\mathrm{PE}^{\prime}(\mathrm{OP} \mid$ anomalous $), t=5.07$ $(p<0.001) ; \mathrm{PE}^{\prime}(\mathrm{OP} \mid$ local $)>\mathrm{PE}^{\prime}(\mathrm{OP} \mid$ anomalous $), t=2.39(p<0.05)$.

Discussion. The overall tendency does not differ from that of analysis 1 . The priming effect $\mathrm{PE}^{\prime}$ is the highest for the global shapes, the lowest for the anomalous shapes, whereas $\mathrm{PE}^{\prime}$ on the local completion lies in between. In figure $11, \mathrm{PE}^{\prime}$ within each stimulus subset is depicted. If one compares the graphs of figures 9 and 11 , the different results on stimulus subset 4 are most noticeable. Note that in case of stimulus subset 4 the number of lines and angles in the visible part of the occluded shape is higher than for any other stimulus subset. In addition the anomalous shape contains very distinctive oblique lines which enhance its salience. The combination of both aspects probably causes the facilitation of the responses on the anomalous shapes, not only after the occlusion prime, but also after the incongruent foreground primes. The present analysis accounts for this. A disadvantage of this analysis, however, is that it does not explicitly test in what way the occlusion prime is completed. Note further that the priming effects according to the present analysis not only depend on just the visible part of the occluded shape (which is the part that the occlusion prime and the incongruent foreground primes have in common), but, more generally, on the perceptual similarity between the incongruent foreground primes and the occlusion prime. The present analysis, therefore, rather accounts for an estimated contribution of the visible part. Nevertheless, the fact that the second analysis leads to the same global ordering of the priming effects of the occlusion prime as analysis 1 , being the highest on the global shapes, the lowest on the anomalous shapes, and intermediate on the local shapes, comprises additional support for this ordering. The clearly different 
results on stimulus subset 4 , however, demonstrate that some caution is necessary when interpreting the analyses.

Within the scope of the present discussion we regard the two analyses as supplementary with respect to each other, without preferring one analysis to the other. Our main point here is that the different priming effects on the global or local shapes on the one hand and the anomalous shapes on the other hand, for both analyses, support the notion that for a given occlusion pattern, both global and local completions are generated by the perceptual system. However, there still is a reservation to be made, which will now be dealt with.

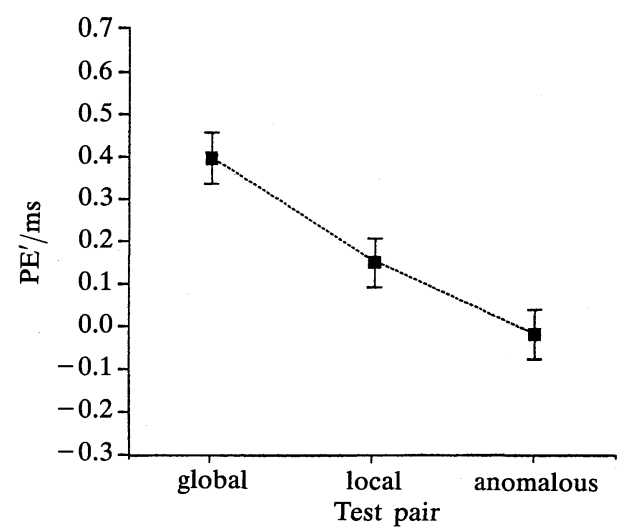

Figure 10. Experiment 1. The mean priming effect, $\mathrm{PE}^{\prime}$, according to analysis 2 .

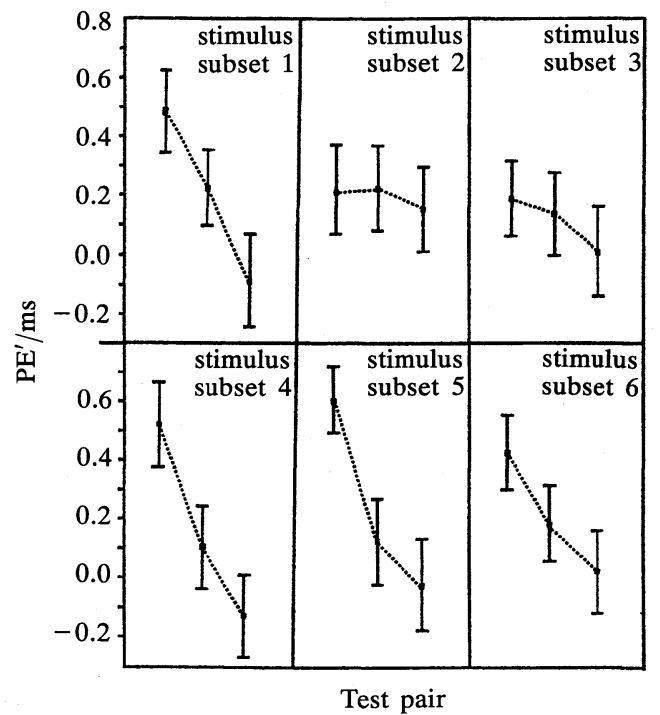

Figure 11. Experiment 1. The priming effect, $\mathrm{PE}^{\prime}$, according to analysis 2 is shown for each stimulus subset. The sequence of test pairs from left to right is: global, local, anomalous.

\section{Experiment 2}

It is possible that, for example, only the global completion is generated. In that case, the priming effect on the local completion might be due to a spreading of the priming effect to completions that are more or less similar to the global completion. This spreading effect may affect the local test pair more than the anomalous test pair.

In order to control for the occurrence of such a spreading effect, a second experiment was set up in which many more anomalous completions were added. These completions ranged by means of small changes from a global completion to a local completion. The rationale behind this experiment setup is that if the priming on the local completion in fact stems from the global completion, the spreading should also affect intermediate completions.

\subsection{Subjects}

Thirty students of the University of Nijmegen, who did not take part in experiment 1 , participated in the experiment. All participants received a small payment.

\subsection{Stimuli and procedure}

The first occlusion pattern of figure 4 was used in the experiment. In the experimental setup, seven different completions were considered. These completions ranged by small changes from global to local; see figure 12. 
In total there were nine different primes: the occlusion prime, seven foreground primes, and the no prime (again represented by two dots). The foreground primes consisted of one of the seven shapes of figure 12 in front of a rectangle. In each trial, one of these primes was followed by one of fourteen test pairs. Seven test pairs consisted of identical shapes, involving the seven completions. The seven other test pairs consisted of nonidentical test shapes, being arbitrary combinations of these completions, but such that if one considers the whole experiment, all test shapes had the same frequency of occurrence. The experimental procedure was the same as in the previous experiment.

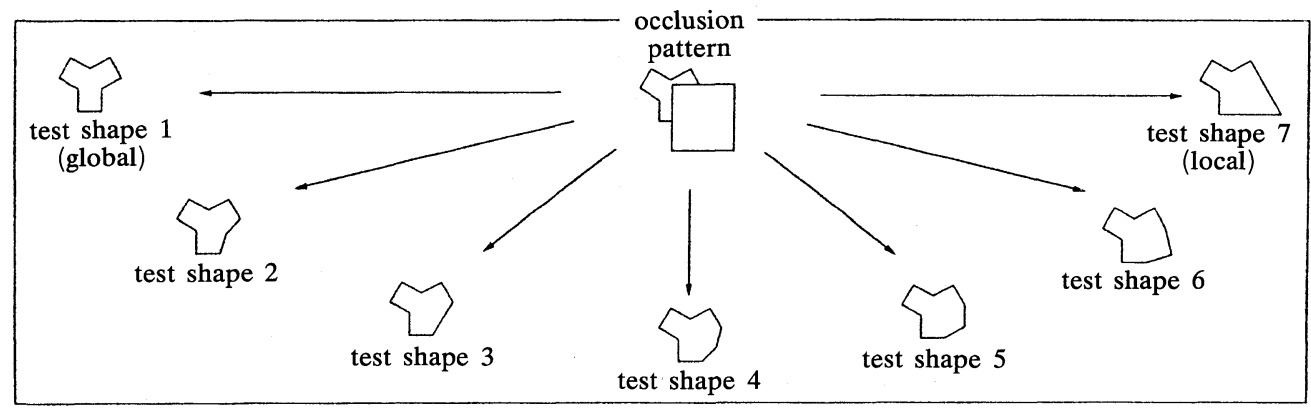

Figure 12. The occlusion prime and test shapes of experiment 2 . The test shapes range by means of small changes from a global completion to a local completion.

\subsection{Results}

In figure 13a, the mean response times and standard errors are shown. As in the previous analysis, only test pairs with identical test shapes are considered. The response times on the incongruent foreground primes for each shape comprise the mean of three subsequent incongruent foreground primes, closest to the test shapes. Only these primes were considered in order to account for the fact that the incongruent foreground primes of the middle test shapes of figure 12 deviate less from the test shape itself, as they are situated around this test shape, than the incongruent foreground primes of the outer test shapes of figure 12 .

\subsection{Analyses}

In figure $13 \mathrm{~b}$ the mean values of the priming effect $\mathrm{PE}(\mathrm{OP} \mid \mathrm{TP})$ according to analysis 1 are shown. These values are relatively high 'near' the global completion, decrease to a minimum at test pair 4, and gradually increase to another local maximum at the local completion. The priming effects of the occlusion prime on both the global completion and the local completion are significantly higher than the priming effect on shape $4(t=1.88, p<0.05$; and $t=1.99, p<0.05$, respectively). Also, the priming effect on shape 2 (the completion nearest to the global completion) is significantly higher than the priming effect on shape $4(t=2.19, p<0.05)$. The priming effect $\mathrm{PE}^{\prime}(\mathrm{OP} \mid \mathrm{TP})$ according to analysis 2 is depicted in figure 13c. According to this definition, the lowest priming effect is achieved for test pair 5. Now, the priming effects of the occlusion prime on the global completion and the local completion are significantly higher than the priming effect on shape $5(t=1.70, p<0.05$; and $t=2.36$, $p<0.05$, respectively). Also, the priming effect on shape 2 appears to be significantly higher than the priming effect on shape $5(t=2.28, p<0.05)$. 


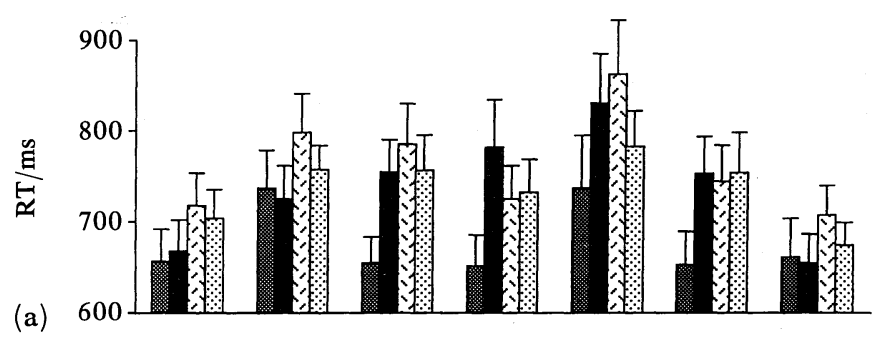

prime type

용 congruent foreground prime

occlusion prime

$\square$ no prime

6 incongruent foreground prime
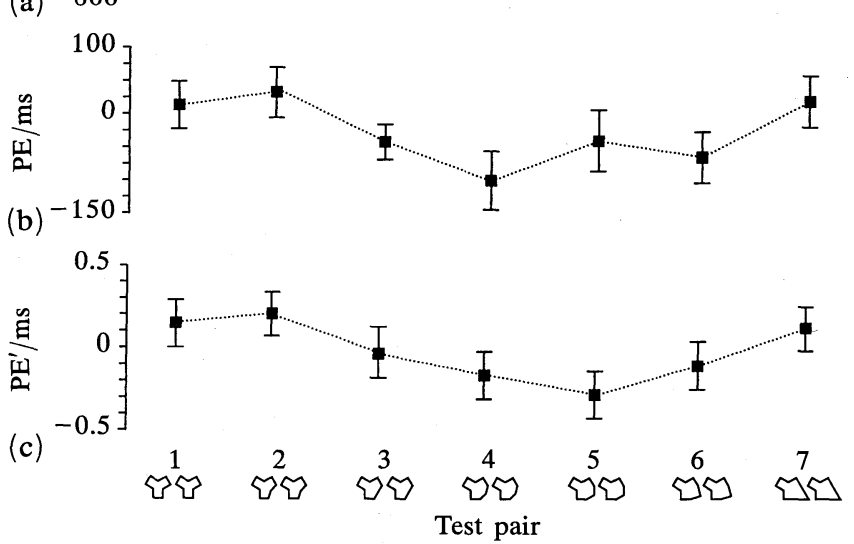

Figure 13. The results of experiment 2. (a) The mean response times (RT) after the various prime types; (b) the priming effects according to definition 1 (PE); (c) the priming effects according to definition $2\left(\mathrm{PE}^{\prime}\right)$.

\subsection{Discussion}

The course of the amounts of priming, with a minimum between the global and local completion, opposes the idea that the priming effect on the local completion is simply due to a spreading of the priming effect caused by the presence of the global completion. The priming effects therefore once more indicate the special status of global and local completions. The relatively high values of the priming effects $\mathrm{PE}$ and $\mathrm{PE}^{\prime}$ on shapes 'near' the global and the local completion (especially shape 2) suggest a spreading of the priming effects from the global and local completions to those nearly global and nearly local completions. Note, however, that the data do not definitely exclude the possibility that those completions are generated as well.

\section{Conclusion}

On the whole, the data support the notion that multiple completions are generated by the visual system. That is, within the present prime duration time of $750 \mathrm{~ms}$ one occlusion pattern might evoke global and local, and perhaps even other, completions. We conclude that, whatever an explanatory model of pattern completion might look like, its output must be characterised by multiplicity rather than singularity.

Acknowledgements. We wish to thank A Sekuler and two anonymous reviewers for their valuable comments on an earlier version of this article. We also thank $C$ Neervoort for his technical assistance. The Netherlands Organization for Scientific Research (NWO) is gratefully acknowledged for funding this project. This research was conducted while Rob J van Lier was supported by a grant from the Foundation for Behavioural Sciences of this organisation (560-262-039). 


\section{References}

Beller H, 1971 "Priming: Effects of advance information on matching" Journal of Experimental Psychology 87 176-182

Boselie F, 1988 "Local versus global minima in visual pattern completion" Perception \& Psychophysics 43 431-445

Boselie F, 1994 "Local and global factors in visual occlusion" Perception 23 517-528

Boselie F, Wouterlood D, 1989 "The minimum principle and visual pattern completion" Psychological Research 51 93-101

Buffart H, Leeuwenberg E, Restle F, 1981 "Coding theory of visual pattern completion" Journal of Experimental Psychology: Human Perception and Performance 7 241-274

Buffart H, Leeuwenberg E, Restle F, 1983 "Analysis of ambiguity in visual pattern completion" Journal of Experimental Psychology: Human Perception and Performance 9 980-1000

Dinnerstein D, Wertheimer M, 1957 "Some determinants of phenomenal overlapping" American Journal of Psychology 70 21-37

Gerbino W, Salmaso D, 1987 "The effect of amodal completion on visual matching" Acta Psychologica 65 25-46

Herbart J, 1850 Lehrbuch zur Psychologie (Leipzig: Voss)

Kanizsa G, 1985 "Seeing and thinking" Acta Psychologica 59 23-33

Kanizsa G, Gerbino W, 1982 "Amodal completion seeing or thinking", in Organization and Representation in Perception Ed. J Beck (London: LEA Publishers) pp 167-190

Kellman P, Shipley T, 1991 "A theory of visual pattern interpolation in object perception" Cognitive Psychology 23 141-221

Lier R van, Helm P van der, Leeuwenberg E, 1994 "Integrating global and local aspects of visual occlusion" Perception 23 883-903

Lier R J van, Helm P A van der, Leeuwenberg E J, 1995 "Competing global and local completions in visual occlusion" Journal of Experimental Psychology: Human Perception and Performance 21 571-583

Mens L, Leeuwenberg E, 1988 "Hidden figures are ever present" Journal of Experimental Psychology: Human Perception and Performance 14 561-571

Rock I, 1983 The Logic of Perception (Cambridge, MA: MIT Press)

Rosch E, 1975a "Cognitive representations of semantic categories" Journal of Experimental Psychology: General 104 192-233

Rosch E, 1975b "The nature of mental codes for color categories" Journal of Experimental Psychology: Human Perception and Performance $1303-322$

Sekuler A, 1994 "Local and global minima in visual completion: effects of symmetry and orientation" Perception 23 529-545

Sekuler A, Palmer S, 1992 "Perception of partly occluded objects: A microgenetic analysis" Journal of Experimental Psychology: General 121 95-111

Sekuler A, Palmer S, Flynn C, 1994 "Local and global processes in visual completion" Psychological Science 5 260-267

Shimaya A, 1994 "A perceptual model of figure segregation and amodal completion" Investigative Ophthalmology and Visual Science, Supplement 351644

Takeishi H, Murakami I, Nakazawa H, Shimojo S, 1993 "Continuity in contour curvature and visual interpolation" Investigative Ophthalmology and Visual Science, Supplement 341084

Wouterlood D, Boselie F, 1992 "A good continuation model of some occlusion phenomena" Psychological Research 54 267-277 